\title{
Conceptual Transfer in English Writing by Chinese Learners of English
}

\author{
WANG Li \\ Qingdao University of Science and Technology, Qingdao, China
}

\begin{abstract}
Language transfer has always been the focus of research in the field of second language acquisition. Conceptual transfer, as the development of language transfer, inspires scholars to explore the first language influence on second language acquisition from a new perspective. Differences between Chinese and English lead to great difficulty to Chinese learners of English. Misuses in syntax may originate from different conceptions between Chinese and English. Mistakes such as sentence localization, prediction relation, word disorder, or sentence with null subject are also prevalent in English compositions. Based on the analysis of language differences between Chinese and English, this paper aims to explore the impact of Chinese on grammar capability in English writing by Chinese learners of English, so as to shed light on English teaching and learning.
\end{abstract}

Keywords: conceptual transfer, Chinese, English writing, grammar

\section{Introduction}

As a cross-language and cross-culture activity, English writing is generally considered as one of the most difficult aspects in learning for Chinese Learners of English (CLEs). They are confused about how to write as well as what to write. They have already gained a profound knowledge in Chinese and the Chinese way of thinking is inveterately rooted when they initiate their study in English. So, CLEs turn to Chinese when confronted with difficulty in English writing. Such difficulties as how to start a sentence, choose a sentence structure, and employ a word make CLEs particularly frustrated.

It is just in these points that learners turn to Chinese in writing an English composition. English and Chinese, however, come from different language families and have distinct characteristics. Chinese belongs to Sino-Tibetan family, while English belongs to Indo-European family. When turning to Chinese for help, CLEs may neglect the differences and thus make many mistakes in the choice of words and syntax. That is to say, their English writing may be interfered and their improvement in writing ability may be hindered by Chinese. This made writing particularly difficult for CLEs.

Language transfer has been the focus of research in the field of second language acquisition. Conceptual transfer, as the development of language transfer, inspires scholars to explore the first language influence on second language acquisition from a new perspective. On the basis of an analysis of language differences between Chinese and English, this paper intends to explore the impact of Chinese on grammar capability in English

WANG Li, lecturer, master, English department, Qingdao University of Science and Technology. 
writing, so as to illuminate the teaching and learning of writing in English .

\section{Literature Review}

Scholars usually explain these phenomenon from the perspective of language transfer. Considerable research has been conducted to delve into problems in second language writing (Cohen \& Brooks-Carson, 2001; Silva, 1993; WANG, 2007). Meanwhile, studies of impact from Chinese occupy a great proportion of research in this field. Influences from thinking mode, culture, and different language features are greatly highlighted in this field.

Since Chinese and English speakers have different thinking mode, some scholars (WANG, 2000; WANG \& WEN, 2002a; WANG \& WEN, 2002b) began to investigate Chinese influence on English writing from the perspective of thinking mode aiming to explore influences in lexical choice, sentence pattern, and structure, and to find ways of how to overcome the transfer to improve writing capability. However, what the previous studies analyzed are differences in thinking mode with no specific explanation on how they affect English writing.

Another central perspective for CLEs' English writing is culture. Cultural differences are sometimes reflected as language differences. QIU and ZHANG (2014) analyzed the common misuses caused by misunderstanding of western culture in English writing. Such papers usually uncover incorrect usages in detail and supply a great resource for the teaching and study of English writing. Nevertheless, this cultural view simply catches the surface mistakes without exploring their root cause.

Some other researchers have contrasted topic prominence Chinese with subject prominence English. These studies approach the internal conscious system and also give an extensive explanatory system. Conceptual transfer from Chinese is inevitable in English writing. Research in this field has been conducted to compare differences in Chinese and English and to further discuss how negative transfer may occur in English writing from morphological and syntactic aspects. It requires that researchers pay close attention to English writing mistakes and explain them in detail. The focus in current conceptual transfer study is to explain the internal conceptual changes from a cognitive linguistics perspective.

\section{Conceptual Transfer}

Conceptual transfer came up with a language transfer mechanism that can predict bidirectional transfer. Conceptual transfer studies hold that a bilingual's conceptual system is a mixed one. It contains the reconstructed conceptual systems from two languages in one container. It is proposed that there are three types of conceptions: L1-based concepts, L2-based concepts and shared concepts. L1 based concepts are those connected to one's first language. These concepts illustrate the concepts that only exist in native language. And similarly, L2 based concepts are those only exist in second language. They are based on the abundant knowledge for the second language. And the shared concepts are those connected with both first and second languages. Regardless of the levels of the language that is used, these three kinds of concepts all have accessibility in language learning. And when L1 based concepts and shared concepts account for most of involved conceptions, first language transfer occurs. And when it impedes second language learning, it is called negative transfer. On the contrary, transfer would be positive if the conceptions involved facilitate learning.

Conceptual transfer observes the internal psychological activity of how first language affects Second 
Language Acquisition (SLA) in conceptions. It tries to explore a wider transfer from deeper levels as cognition and conception. Conceptual transfer also emphasizes how second language is changed as transfer constantly occurs. According to the understanding of language acquisition, one's conceptual reconstruction gets deeper as their second language learning experience accumulates. However, sometimes it ignores conceptual features in first language, which plays an important role in conceptual transfer.

As for CLEs, they understand and remember English with the help of Chinese at the beginning stage. Thus beyond all doubt, Chinese grammar affects English learning to some extent.

\section{Differences Between Chinese and English}

Study is to construct the new knowledge on the basis of the old one. It is a process to understand, digest, assimilate, and master the new one by taking advantage of the old one. Thus during English grammar study, similarities between these two grammar knowledge will boost the understanding of English grammar. A comparison between Chinese and English grammar reveals that differences between English and Chinese are a fundamental cause of conceptual transfer. Knowing the differences is crucial for analyzing mistakes. The following generalizes some basic differences in language structure between Chinese and English.

(1) In English, tense is an independent constitution. The main verb has to combine with tense. And because an English sentence can only contain a main verb, other verbs have to change into another form to cater the main verb and the sentence. Yet tense does not exist in Chinese and many verbs can co-occur in one Chinese sentence;

(2) In English, tense has to be in accordance with subject, which means English needs case in grammar. Null subject it is a special yet typical example. However, subject grammar is not that strict in Chinese;

(3) English syntax grammar has another inclination to put modification after the verb. Extraposition is one of the most important one.

\section{Syntactic Transfer}

\section{Sentence Localization}

"The price is expensive, the content is hard to understand, the novel that Tom wrote, the ordinary bookstore does not sell (价格很贵，内容又难懂，汤姆写的那本书普通书店不卖)”.

Sentences like the above one are commonly found in the compositions of CLEs. Actually, these series of English sentences are a translated version from the Chinese topic-crucial sentence in the bracket. This is an important contrast between English and Chinese sentence. CLEs directly transferred the Chinese concepts into English when facing difficulties in writing.

The topic of this sentence has three crucial clauses. Besides, as the topic, "the novel that Tom wrote" cannot be simply placed at the initial part if we do not change the language. Therefore, the topic and the comments are only loosely connected. There are other mistakes that may be found violating English grammar in this sentence. "The novel that Tom wrote", as the topic noun phrase, does not have a fixed predicate. Meanwhile, no clear causality connection is displayed and no conjunction is found to indicate the causality of two arguments among different parts of the sentence.

Compared with Chinese, English is a subject-oriented language while Chinese is a topic-oriented language. Chinese sentences usually begin with a topic or theme and are usually followed by some comments or statements. 
Besides, predication relationship is not necessary in Chinese for the topic/theme and comments/statements. A typical English sentence, however, has to live up to some forms. First, a sentence has to possess a subject and a predicate. And this predicate also contains the main verb of the sentence. Neither lack of predicate nor subject is allowed in English. Moreover, if more than one verb is presented in an English sentence, all the other verbs except for the main verb (predicate) have to change their forms. The infinitive and present or past participle form are all acceptable. From this point, the sentence should be revised as follows:

(1) Regarding the novel that Tom wrote, its price is expensive, and its content is hard to understand, thus the ordinary bookstores do not sell it;

(2) The novel that Tom wrote is expensive. Also, its content is hard to understand. So the ordinary bookstores do not sell it.

\section{Prediction Relation}

Compared with Chinese sentences, English sentences are more confined to grammar forms. And one important reason is that English sentences are based on prediction relation. Prediction relation refers to the semantic match relation between a noun/noun phrase and its predicate. This law is strictly followed in English while is not that important for Chinese.

Generally speaking, $q i$ (骑) in Chinese is a transitive verb. Thus it needs a subject to indicate the actor and also needs an object to indicate the thing that is ridden. Yet in Chinese, the behavioral verb can usually be followed by a non-actor as subjects (such as place and scope), or as object (such as tool, place, and time). CLEs, influenced by Chinese, may write sentences that are definitely unacceptable in English.

(1) On the road is riding a bike (公路上骑着一辆自行车 (the subject is the place)).

(2) This bike drives John to sweat all over her body (这辆自行车骑得琼浑身是汗 (the subject is the person who is actuated)).

(3) This going back to work need two bikes (这次回去上班得用两辆自行车 (he subject is the scope)).

The fact that English always follow subject-predicate relation strictly makes these sentences wrong. In other words, ride is a verb referring to the action of operating a bicycle. Therefore a subject must be presented as the actor and so does the object to be an effector. If CLEs arrange the sentences according to Chinese grammar, the ride sentence cannot comply with the English grammar.

\section{Word Order and Logic}

Semantic predication in a sentence is reflected in two parts with one part semantically matching or modifying the other. The clausal head is known for verbal argument (for example subject or object) and the other one is like adjective modifying noun or adverb modifying verb. The point is that the modification usually has its directivity (Cumming, 1987; Kroll, 1993; Zamel, 1996). Chinese predication modification is usually from left to right (clausal is in the left side to the noun) whereas English predication modification has an adverse direction. This difference, however, is far more interesting than simply a difference in word order. Chinese often use several short sentences to express the idea which can be expressed just in one single English sentence. Consequently, CLEs tend to write sentences like:

"A government should be dedicated to the people because some people sacrificed much for the country and they devoted their youth or even lives to it. In fact, they may devote it to their beloved ones. So, the government 
must pay the greatest respect to them".

This series of sentences just reveal the Chinese way of thinking and can be regarded as a liberal equivalent to the Chinese version. The series of the sentences can be revised into the following:

"A government that is truly dedicated to the people must pay the greatest respect to those who have sacrificed much for the country, to which they may have devoted their youth or even lives, which they could otherwise have devoted to their beloved ones".

We noted that since complicated modification appears usually to the right of the noun in English, the predication also extend from right to left and may form into a recursive modification like the revised sentence (Billy, 2002). CLEs capability in English writing will be hindered if they rely on Chinese and Chinese way of thinking by transferring the Chinese structures and concepts directly to English.

\section{Sentence With Null Subject}

An additional thing need to be stressed is the null subject $i t$. As a placeholder, it conveys nothing in meaning and its place will not be restricted by semantic meaning. Thus it is quite flexible about its place. Here extraposition is a typical example. Extraposition means the word order of a sentence is arranged in such a manner that a relatively "heavy" constituent appears to the right of its canonical position. However, the null subject does not exist in Chinese. They will produce such English equivalent of “人们认为地球是围绕着太阳转的” as "people think that the earth moves around the sun", instead of generating sentences like "it is generally assumed/believed/that the earth moves around the sun" or "it has been argued/proposed/questioned that...the earth moves around the sun".

This kind of sentence pattern in English has two features. First, they all use extraposition and the null subject $i t$. Secondly, the verb is used in passive voice. What makes extraposition possible in English is the null subject it. We can infer from above that extraposition will not be possible without it as a null subject to reach agreement. And that also makes it a special sentence pattern in English. For CLEs, both of these two features are absent in Chinese grammar. What they often unconsciously do is creating an English sentence by transferring the Chinese grammar into English.

\section{Conclusion}

Systematic and distinct grammar conceptual transfers are revealed in this study. We found conceptual transfer in sentence localization, prediction relation, word order, and logic and sentences with null subject. By studying some frequently misused grammar, it is clear that transfer occurs in thinking mode and conceptual scope because the knowledge of Chinese grammar affects English writing. These transfers lead to some Chinese way of thinking in English expressions and impede second language acquisition. Our original way of thinking and conceptual scope system will adjust or change by getting in touch with English for a long time or improving some linguistic knowledge. Teachers need to help students during the learning process and help students cast off the negative transfer problems to some extent.

Language transfer is full of changeability because it is only a subsystem of interlanguage development, which indicates the fundamental meaning of dynamic system. Therefore, it is important to combine the dynamic theory with transfer theory in second language acquisition. Future research should pay attention to the transfer in the non-linguistic process, the long term development and the micro change in short term. 


\section{References}

Billy, W. (2002). Language-switching: Using the first language while writing in a second Language. Journal of Second Language Writing, 1(11), 7-28.

Cohen, A. D., \& Brooks-Carson, A. (2001). Research on direct vs. translated writing: Students' strategies and their results. The Madern Language Journal, 85(2), 169-187.

Cumming, A. (1987). Decision making and text representation in ESL writing performance. Paper presented at The 21st Annual TESOL Convention, Miami.

Kroll, B. (2003). Introduction: Teaching the next generation of second language writers. In B. Kroll (Ed.), Exploring the dynamics of second language writing (pp. 1-10). Cambridge: Cambridge University Press.

QIU, G. Z., \& ZHANG, H. J. (2014). Investigation and analysis of present English major writing course. Journal of Ningbo Radio \& TV University, 2(3), 88-92.

Silva, T. (1993). Toward an understanding of the distinct nature of L2 writing: The ESL research and its implications. TESOL Quarterly, 27, 57-77.

WANG, J. J. (2007). Review of studies on L2 writing processes: Cognitive and psychological perspectives. Foreign Language World, 5, 2-9.

WANG, W. Y. (2000). An investigation into Ll use in the writing process of tertiary-level EFL Learners in China (Unpublished Ph. D. Dissertation, Nanjing University).

WANG, W. Y., \& WEN, Q. F. (2002a). An investigation into L1 use in the L2 writing process of tertiary-level English learners in China. Journal of PLA University of Foreign Languages, 25(4), 64-67.

WANG, W. Y., \& WEN, Q. F. (2002b). The influence of L1 thinking on L2 writing. Foreign Languages and Their Teaching, 10, 18.

Zamel, V. (1996). Teaching composition in the ESL classroom: What we can learn from the research in the teaching of English. TESOL Quarterly, 10(1), 67-76. 